\title{
The most problematic symptoms of prion disease - an analysis of carer experiences
}

\section{Liz Ford, ${ }^{1}$ Peter Rudge, ${ }^{1,2}$ Kathy Robinson, ${ }^{3}$ John Collinge, ${ }^{1,2}$ Michele Gorham, ${ }^{1}$ and Simon Mead ${ }^{1,2}$}

\author{
${ }^{1}$ NHS National Prion Clinic, National Hospital for Neurology and Neurosurgery, University College London (UCL) Hospitals NHS Foundation Trust, \\ London, UK \\ ${ }^{2} M R C$ Prion Unit at UCL, Institute of Prion Diseases, UCL, London, UK \\ ${ }^{3}$ The Open University, Milton Keynes, UK
}

Objectives: Prion diseases are rare dementias that most commonly occur sporadically, but can be inherited or acquired, and for which there is no cure. We sought to understand which prion disease symptoms are most problematic for carers, to inform the development of outcome measures.

Design: Self-completed questionnaire with follow-up of a subset of participants by structured interview.

Setting: A nested study in the UK National Prion Monitoring Cohort, a longitudinal observational study.

Participants and measurements: 71 carers, of people with different prion diseases with a wide range of disease severity, identified 236 of their four most problematic symptoms by questionnaire which were grouped into ten domains. Structured interviews were then done to qualitatively explore these experiences. Eleven family carers of people with prion disease were selected, including those representative of a range of demographics and disease subtypes and those who cared for people with prion disease, living or recently deceased. Interviews were transcribed and formally studied.

Results: The six most problematic symptom domains were: mobility and coordination; mood and behavior; personal care and continence; eating and swallowing; communication; and cognition and memory. The prevalence of these symptoms varied significantly by disease stage and type. A formal analysis of structured interviews to explore these domains is reported.

Conclusions: We make suggestions about how healthcare professionals can focus their support for people with prion disease. Clinical trials that aim to generate evidence regarding therapies that might confer meaningful benefits to carers should consider including outcome measures that monitor the symptomatic domains we have identified as problematic.

Key words: CJD, Creutzfeldt-Jakob, prion, carer, caregiver, burden

\section{Background}

The care of a person with dementia can have an overwhelming impact on family carers: carer burden, stress, depression, physical illness, and decreased quality of life, are all reported as results of caring (Livingston et al., 2017). Prion diseases are a rare and varied group of neurodegenerative dementias caused by the misfolding of a normal body protein.

Correspondence should be addressed to: Simon Mead, NHS National Prion Clinic, National Hospital for Neurology and Neurosurgery, University College London (UCL) Hospitals NHS Foundation Trust, London, UK. Phone: 0044 (0) 207679 5152. Email: s.mead@ucl.ac.uk. Received 27 Jun 2018; revision requested 19 Jul 2018; revised version received 06 Aug 2018; accepted 10 Aug 2018. First published online 24 October 2018.
Occurring worldwide, sporadic Creutzfeldt-Jakob disease (sCJD) has no known cause; inherited prion disease (IPD) is caused by genetic mutation of the prion protein gene; iatrogenic Creutzfeldt-Jakob disease (iCJD) and variant Creutzfeldt-Jakob disease (vCJD) are acquired through medical procedures or diet (Collinge, 2001). They are heterogeneous in presentation, but symptoms typically progress rapidly and include cognitive deficits, ataxia, apraxia, dysarthria, dysphagia, mood swings, altered sleep patterns, hallucinations, and myoclonus. There are no effective treatments that modify the underlying course of prion disease (Trevitt and Collinge, 2006).

Relative to common dementias, carer burden in prion disease is severe when studied with the Zarit 
Burden Interview (ZBI), Neuropsychiatric Inventory (NPI-Q), and other assessment instruments (Uflacker et al., 2015). Other than this single study, which aimed to compare atypical dementias and involved seven patients with sCJD, the subject area has not yet been researched. Recent work in typical dementia syndromes, such as the investigation of an optimal time to move to a care home (Cole et al., 2018) and use of non-pharmacological interventions in dementia care (Cohen-Mansfield, 2018), may be very relevant to the care of people with prion disease. In order to start to develop a scientific interest in the care of persons with prion disease, we planned descriptive research as part of a longitudinal observational cohort study in the UK.

The National Prion Monitoring Cohort (NPMC) was launched in 2008 with the aims of monitoring disease progression, development of rating scales, and providing a knowledge base for future trials (Thompson et al., 2013). Many dementia progression scales were found to have a floor effect in prion disease (Mead et al., 2011). A new prion disease rating scale comprising of questions from two functionally oriented scales was developed (Thompson et al., 2013). Progression scales in dementia have been criticized for not accurately reflecting the problems caused by functional deficits as perceived by carers, or developed based on clinicians' intuition (Bucks et al., 1996; Nasreddine et al., 2005). Rating scales should measure items that are universal to the population of interest, and the target population should be involved in the development to judge the relevance and comprehensiveness of scale items (Bucks et al., 1996; Pfeffer et al., 1982; Sikkes et al., 2009; Terwee et al., 2007). Therefore, capturing carers' views is essential. It is also imperative to gain an insight into a carer's perspective, so appropriate care and support can be provided.

In this paper, we describe the informal carer's experience of looking after people with prion disease with particular reference to those symptoms that were of most concern to them comprising physical, behavioral, and cognitive dysfunction. While prion diseases are a rare cause of dementia, they exhibit the whole range of management problems found in varying proportion in the more frequently encountered neurodegenerative dementias and movement disorders, such as Alzheimer's disease, Cortical Lewy body disease, progressive supranuclear palsy, and frontotemporal dementias. Care of people with prion disease has not been extensively studied. This is, therefore, an ideal disease group to investigate the most troubling symptoms for carers to manage and discuss some practical solutions we have identified together.

\section{Method}

\section{Site, consent and ethics}

The National Prion Clinic (NPC) is a national referral center for prion disease and is funded by the NHS to provide diagnosis and care for people with or at risk of CJD. The clinic is integrally linked with the MRC Prion Unit at UCL at the Institute of Prion Diseases. Every person with prion disease is allocated a specialist nurse to provide support and advice to them and their family, assist with coordinating local care, and give guidance and training to care staff.

Informed consent was obtained directly from study participants or their next of kin. Ethical approval for the NPMC was obtained from the Scotland A Research Ethics Committee. The nested study reported here was entered as an amendment to the original protocol and approved by the Scotland A Research Ethics sub-committee on $28^{\text {th }}$ March 2011.

Participants were approached over the telephone or during a clinical visit. Aims of the study were explained on approach and at interview. Informed consent was obtained. All were informed participation was voluntary, they could withdraw at any time, and that interviews could be paused or terminated if they became upset or did not wish to continue. Participants could also decline to answer specific questions. All participants were given contact details for clinic staff if they needed to discuss issues raised in the interview. Five interviews were paused due to interviewee becoming upset, but all chose to continue. There were no withdrawals from the nested study, and no participants needed to contact clinic staff for support post interview. Participants were given a code and this code was used on all tapes and transcripts. Only the primary researcher, who conducted the interviews, could identify participants from the codes.

\section{Interviewer administered questionnaires}

Between 2009 and 2012 during Cohort Study visits, informal carers were asked to complete a questionnaire-based assessment of disease impact. All participants were non-professional, namely spouses, children, parents, partners, and so forth, and were required to be the principle informal provider of care for the person with prion disease; we did not require a specific time spent caring per week for eligibility. We asked, "Which are the four biggest problems for you and/or other caregivers?" Carers were instructed to include all problems, including mood, attitude, and general behavior. More than one symptom could be listed, but carers were asked to rank those most problematic first, and indicate how troubling these were on a scale from 
Table 1. Verbatim examples of the six most problematic symptom domains

\begin{tabular}{lcc}
\hline SYMPTOMATIC & TIMES & \\
AREA & MENTIONED & EXAMPLES
\end{tabular}

Mobility, balance, praxis

Personal care, continence

Behavior, hallucinations

Eating, swallow, secretions
A "Cause she, she did have a fall at one point and she, um. It was when I wasn't there, um and she'd, she fell over and she broke two of her ribs. And, and we didn't know she'd even fallen for three days. She didn't tell us, and then ... she'd was, breathing, was getting bad and she ended up going into hospital for that"

B "having all kinds of difficulties with care agency ... saying that carers aren't meant to, aren't meant to take weight, weight bear. Which is ridiculous, it's not like they have to pick him up? But they have to stand him on his feet and transfer him on to another, chair and the carers are happy to do it, but the care agency isn't happy for them ... to do it, so. It just makes, you know, life difficult"

C "more recently he's been having difficulty, well he was having difficulty lifting a coffee cup ... that has really limited us, because, you know, he finds it awkward and embarrassing"

D “As Mum got quite poorly, you know, the speech therapist was going 'well, we'll bring these cards round' and they brought us round some sheets with like letter A on one letter, well she can't bloody point to them, 'cause her arms were all over the place, so they're no good"

E "Um, and I do have to go in the loo afterwards, if I can, and just check that it's all clean and lovely"

F "And she was wondering why people were coming in. Especially when it, like, came to her having a shower or a bath"

G 'So she wanted to talk, just couldn't understand her. Um. That's a huge, huge regret that I have no idea what the last thing she, the last thing she said to me was"

$\mathrm{H}$ "She kept her faculties longer than she kept her voice"

I "yeah, if he'd had enough food and that you knew or if he didn't like it you knew"

J "going on the train it had to be the same train, it had, he had to stand at the same place on the platform. he would get very distressed if there was err an issue with the train or if I wasn't there to pick him up his rucksack had to be in a certain place, his shoes had to be in a certain place in the room"

$\mathrm{K}$ "And because of that and because of the way he was just working so much all the time, I sort of lost the plot with him one night and accused him of having an affair [laughs]. You know, I thought that was, like he'd been in [name of town] and then saying he wasn't there, just all didn't look good, so I accused him of having an affair which he denied"

$\mathrm{L}$ "But she could be really nice as well in between the, the violent ... episodes. She could have times when she was really nice to be with still. That's probably why you wanted to keep her at home, wasn't it, 'cause she was still nice"

$M$ “Er, she certainly talked to people who weren't, who we couldn't see"

$\mathrm{N}$ "and he will shy away from foods that he finds difficult to eat. Don't like beans 'cause they're hard to eat, um"

$\mathrm{O}$ "and of course he choked eventually. Um. You know, because that was one of the things they warned us about that um. Choking. And he did".

P 'I wasn't here this day, but he was off, but he was going to have the girls. They were over at my Mum and Dad's cause he had something to do here, working on a car or something. He went back over, got the girls, brought them back over here. And 5 minutes later he went back over my Mum and Dad's and said, "I'm here to get there girls". He'd completely forgotten that he'd already picked them up"

$\mathrm{Q}$ "But, by the time the dig, the official diagnosis came out [she] was not in any state to, yeah, she'd already forgotten she was ill" 
Table 2. Demographic details of the interviewed carers

\begin{tabular}{|c|c|c|c|c|c|c|c|}
\hline PARTICIPANT & AGE & $\begin{array}{l}\text { DIAGNOSIS OF } \\
\text { RELATIVE }\end{array}$ & $\begin{array}{l}\text { RELATIONSHIP } \\
\text { TO RELATIVE }\end{array}$ & Јов & ETHNICITY & GENDER & $\begin{array}{l}\text { STATUS OF } \\
\text { RELATIVE }\end{array}$ \\
\hline KX01 & 52 & IPD & Wife & Teacher & White British & Female & Alive \\
\hline DX02 & 43 & IPD & Partner & Unemployed & White British & Female & Alive \\
\hline DX03 & 40 & sCJD & Wife & Office Worker & White British & Female & $\begin{array}{l}\text { Deceased, } \\
10 \text { months }\end{array}$ \\
\hline DX04 & 64 & IPD & Husband & Builder & White British & Male & Alive \\
\hline SX05 & 50 & iCJD & Wife & Housewife & White Irish & Female & Alive \\
\hline BX06 & & sCJD & Partner & Self Employed & White British & Male & Alive \\
\hline SX07 & 65 & sCJD & Wife & Housewife & White British & Female & $\begin{array}{l}\text { Deceased, } \\
4 \text { years, } 1 \text { month }\end{array}$ \\
\hline CX08 & 69 & sCJD & Husband & Self Employed & White British & Male & Deceased, 1 year \\
\hline JX09a & 48 & vCJD & Father & Self employed & White British & Male & $\begin{array}{l}\text { Deceased } 4 \text { years, } \\
9 \text { months }\end{array}$ \\
\hline JX09a & & vCJD & Mother & $\begin{array}{l}\text { Airline } \\
\text { Stewardess }\end{array}$ & White British & Female & $\begin{array}{l}\text { Deceased } 4 \text { years, } \\
9 \text { months }\end{array}$ \\
\hline PX10 & 42 & IPD & Son & Manager & White British & Male & $\begin{array}{l}\text { Deceased, } 4 \text { years, } \\
1 \text { month }\end{array}$ \\
\hline
\end{tabular}

Note: IPD = Inherited Prion Disease, sCJD = Sporadic CJD, vCJD = Variant CJD, iCJD - Iatrogenic CJD.

"not troubling at all" to "severely troubling." Seventy-one questionnaires were completed, listing between one and four symptoms. The symptom names were collated by a doctor and nurse from the NPC team and grouped into 10 symptomatic domains (Table 1, Chart 1). Participants were consulted in a variety of settings $(65 \%$ home, $17 \%$ short admission to hospital for diagnosis, $15 \%$ nursing home, and $2 \%$ hospice). In the non-home setting, the perspective requested was from the provision of care at home. Informed by the questionnaires, an inductive qualitative research study was designed with the aim of gathering information about the carers' experience of the symptoms of prion disease and the implications for care (Clarke and Braun, 2017; Lin et al., 2012).

\section{Sample}

A purposeful sampling technique was used to recruit 11 carers of 10 people with prion disease; one interview was carried out with both parents of a person with vCJD (Greenhalgh and Taylor, 1997; Higginbottom, 2004; Tuckett, 2004). The only inclusion criteria were that participants were carers of people with prion disease enrolled in the NPMC (Table 2). Excluding people with prion disease not enrolled in the NPMC was not a source of bias as the enrollment rate was $98 \%$ of all people diagnosed with probable CJD seen by the NPC in the UK at the time of the study. Five carers were caring for people with prion disease at the time of interview; five were interviewed after the person with prion disease had died, in order to capture the entire disease duration. Participants came from a range of locations and socioeconomic backgrounds. All participants were White British or White Irish, as were $95.5 \%$ of NMPC participants.

\section{Interviews}

All participants were interviewed on their own to maintain privacy and given a choice of venue (Elmir et al., 2011; Walker, 2011). Five interviews were conducted in the participant's home, and five were conducted while the carer was attending the NPC for another reason (no carers were excluded due to travel restrictions) (Tuckett, 2004). Interviews at the NPC were conducted in a private room with no interruptions (Elmir et al., 2011; Walker, 2011). All participants were interviewed by the same interviewer, an experienced prion disease nurse, to ensure consistency.

Participants were asked to describe the first symptoms or changes they noticed, then discuss each symptom or change in condition in chronological order, describing how they had felt, what impact this had on their life, and their ability to provide care. A typical opening question would be "Thinking back, can you describe the first symptoms or change you noticed?" The interview carried on until the disease progression had reached the current time, or all symptoms until death had been discussed. If a symptom from one of the defined 10 symptomatic areas had not been spontaneously mentioned, the interviewer asked a direct question, such as "Do you think X ever experienced hallucinations?" in order that all possible symptoms had been considered. Clarifying questions with words like "such as" and "checking back" were used to 
Table 3. Frequencies of subtypes of symptomatic areas

\begin{tabular}{|c|c|c|c|}
\hline SYMPTOMATIC AREA & CODES WITHIN AREA & NO. EXCERPTS & TOTAL \\
\hline \multirow[t]{4}{*}{ Mobility, balance, praxis } & Coordination & 44 & 134 \\
\hline & Mobility & 76 & \\
\hline & Wandering & 5 & \\
\hline & Writing & 9 & \\
\hline \multirow[t]{2}{*}{ Personal care, continence } & Continence & 21 & 33 \\
\hline & Personal care & 12 & \\
\hline \multirow[t]{3}{*}{ Communication } & Communication & 7 & 57 \\
\hline & Speech & 41 & \\
\hline & Writing & 9 & \\
\hline \multirow[t]{5}{*}{ Behavior, hallucinations } & Behavior & 72 & 206 \\
\hline & Hallucinations & 12 & \\
\hline & Mood & 71 & \\
\hline & Social preservation & 11 & \\
\hline & Personality & 40 & \\
\hline \multirow[t]{2}{*}{ Eating, swallowing, secretions } & Eating and swallowing & 41 & 55 \\
\hline & Food preference & 14 & \\
\hline \multirow[t]{6}{*}{ Cognition, memory, navigation } & Concentration & 6 & 149 \\
\hline & Confusion & 19 & \\
\hline & Memory & 45 & \\
\hline & Navigation & 12 & \\
\hline & Cognitive problems & 46 & \\
\hline & Recognition & 21 & \\
\hline Sleep patterns, drowsiness & Sleep & 34 & 34 \\
\hline Myoclonus, seizures & Myoclonus, seizures & 9 & 9 \\
\hline \multirow[t]{2}{*}{ Rigidity, tissue viability } & Stiffness & 2 & 9 \\
\hline & Speed & 7 & \\
\hline Pain, sensory symptoms & Pain & 16 & 16 \\
\hline \multirow[t]{4}{*}{ Other } & Loss of consciousness & 1 & 13 \\
\hline & Sex & 5 & \\
\hline & Vision & 2 & \\
\hline & Terminal phase & 5 & \\
\hline
\end{tabular}

ensure accuracy. At the end of the interview, the participant was asked what, if anything, they felt would have made a positive difference to their experience as a carer. Interviews were recorded and transcribed; four by the interviewer and six by an experienced medical secretary. All transcripts were read and reread several times and checked back against the recording by the interviewer for accuracy (Clarke and Braun, 2017).

\section{Data analysis}

NVivo 9 software (QSR International Inc.) was used to assist with data management. Topics were assigned codes. Each transcript was coded independently using the codes found in the first and previous transcripts. The first level of analysis coded for symptoms, which were spontaneously mentioned, were those given in response to questioning and care and relationship issues. In the second level of analysis, all excerpts coded under symptoms were recoded for specific symptoms, such as "memory" or "use of tools" (Table 3).
These were not defined by the 10 symptomatic areas but based on the participants own words. All primary coding was performed by the interviewer, as they were fully immersed in the data and had an understanding of how the participant had expressed themselves. To establish accuracy and validity, random excerpts were independently coded by two additional prion disease nurses to seek consensus. Once each specific symptom had been coded, excerpts were analyzed to look for common themes based on commonly expressed symptoms.

\section{Results}

The majority of questionnaires were completed by the carers of people with sCJD (50\%) with the remainder mostly IPD (40\%). There were 236 symptoms identified, ranked, and classified into 10 domains by the study team (Figure 1). The frequency with which carers identified symptoms in different domains 

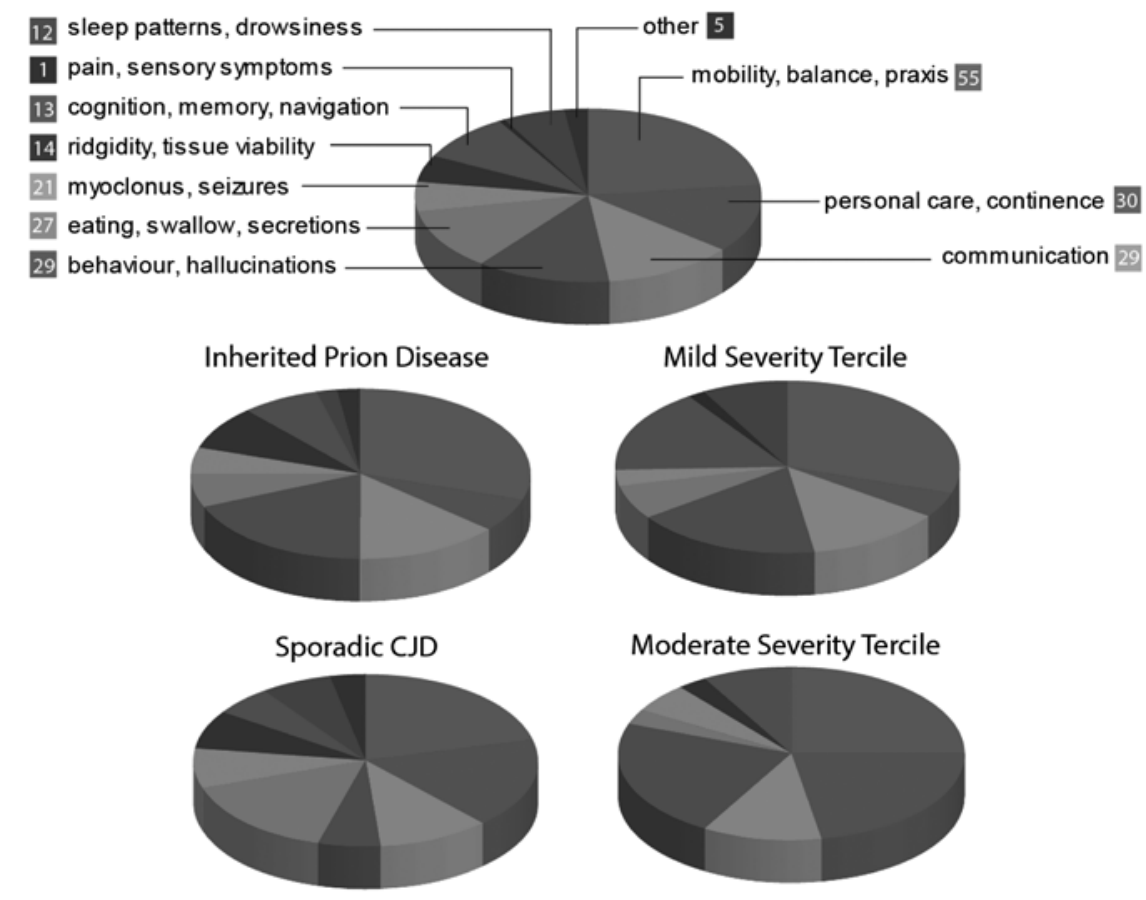

Moderate Severity Tercile

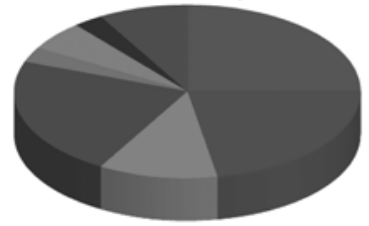

Most Severe Tercile

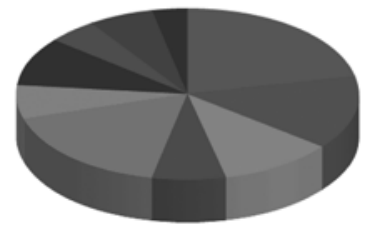

Figure 1. Pie charts illustrating the proportion of symptomatic domains identified by carers as significant (top, 71 carers of all types of prion disease). Below, we show these data split into disease type (left) and disease severity (right). These subgroups showed statistically significantly different responses (see results).

varied significantly by disease type (sCJD vs IPD, Chi-squared test, $p=0.007$, Figure 1) and disease severity (mild, moderate, or severe terciles based on MRC Scale score, Chi-squared test, $p=4 \times 10^{-6}$, Figure 1). Age and sex of the carer and the relationship to the person with prion disease did not impact the symptoms reported. Due to the heterogeneous nature of prion disease, carers described a wide range of symptoms. The six symptomatic areas most commonly mentioned were selected for discussion: mobility and coordination; continence and personal care; communication; mood and behavior; eating and swallow; and cognition and memory (Table 1, Figure 1). In sCJD, relative to IPD, carers were more likely to identify problems with feeding and personal care and less likely to identify behavioral and mobility issues. As the disease became more severe, carers were more likely to identify feeding, myoclonus, and sleep disorder as problematic, whereas in the early stages the more significant problems were behavior and mobility, consistent with previous work (Thompson et al., 2014, 2013).

\section{Mobility and coordination}

Prion diseases cause movement disorders. In some cases, this was the predominant early feature that lead to diagnosis. This could manifest itself into problems with leisure activities, such as tennis, and work related skills, such as writing. Problems with mobility were frequently cited as a cause of hazards and had an impact on the care environment (Table 1, A) and on interactions with care staff (Table 1, B). Mobility and coordination worsened as the disease progressed in all cases, leading to a loss of independence for people with prion disease and carers and a restriction of social activities. Also, all carers reported problems with the affected person using a knife and fork, which ultimately led to the person needing to be fed. This had a profound effect on one family (Table 1, C). Poor coordination also limited the usefulness of standard communication aids, in some cases rendering them useless, causing added frustration (Table 1, D). 


\section{Continence and personal care}

Incontinence was a complex problem and not simply a matter of physical control. People were incontinent due to a lack of awareness or delay in accessing toilet facilities due to their environment or physical problems with mobility or clothing. There were many care needs that a regular continence service wasn't able to meet. Particularly problematic were people in the mid-stage of the disease, who were continent and could take themselves to the toilet, but couldn't properly clean themselves or the bathroom afterward (Table 1, E). People with prion disease often initially needed assistance with washing due to balance or cognitive problems, then, over time, lose the ability to wash themselves completely for a combination of reasons (Table 1, F).

\section{Communication}

Communication became more and more problematic as the disease progressed. In the early stages, the affected person tended to find it easier to speak to one person at a time and found group conversations difficult to follow. Several carers reported difficulties understanding the person's needs because of slurred speech, which caused frustration for the affected person (Table 1, G). Standard communication aids were ineffective due to poor limb coordination and cognitive problems. Talking on the phone was cited as particularly difficult and was often a problem in the early stages of the disease, possibly due to the lack of visual cues and nonverbal signs. Although slurring was mentioned, many carers stated that the affected person simply talked less and less, would sit in silence rather than initiate conversation. Carers felt that people with prion disease chose not to speak any more, despite retaining understanding of what was said to them (Table $1, \mathrm{H}$ ), but were able to communicate their wishes into advanced stage of disease (Table 1, I).

\section{Mood and behavior}

Many carers reported subtle changes in behavior or routine as an early, pre-diagnosis symptom. This often took the form of obsessive, paranoid, or confused behavior (Table 1, J, K). Aggressive outbursts were often the result of frustration. Affected people were often low in mood and became withdrawn. Despite the changes in mood and behavior, carers often reported that people with prion disease did retain some social skills such that they were able to make more of an effort when others were around and could distinguish between home and other environments. Also, these changes in mood and behavior would fluctuate (Table 1, L). Hallucinations were a feature for some people with prion disease. Carers reported people with prion disease believing things they'd seen on television being real or would interact with hallucinations (Table 1, M).

\section{Eating and swallowing}

Weight loss was reported as an early sign by several carers, followed by slower eating and chewing, which was a common phenomenon when people were moderately affected. Sometimes there was an insight into difficulties with people avoiding foods that were found difficult to manipulate in the mouth (Table 1, N). Swallowing problems were a common symptom of the moderate and advanced stages of prion disease. This would manifest itself initially as coughing during eating then choking (Table 1, O).

\section{Cognition and memory}

As expected with a disease causing dementia, carers frequently reported a range of cognitive problems, which were often a trigger for seeking medical review. Short-term memory problems were an early sign, and mistakes could be hazardous and had a potentially disastrous impact on family life (Table 1, P). Confusion was particularly bad when people woke during the night, and cognitive problems increased as the disease progressed. Problems with navigation while driving were mentioned as an early sign and were a trigger for intervention and also noted in very familiar environments later in the disease course. Due to the rapidity of prion disease progression, formal diagnosis often came too late for the affected person to understand (Table 1, Q).

\section{Discussion}

The aim of this descriptive study was to explore the experiences of carers of people with prion disease, to determine which symptoms were most problematic and to discuss these data around improvements in management and outcome measures. We hypothesized that carers of people with prion disease would identify a consistent set of troublesome symptoms by questionnaire, and these would show some predictable differences between disease types and severities, which were explored further.

The six of the most troubling symptomatic areas for carers were: mobility and coordination, continence and personal care, communication, eating and swallowing, behavior and hallucinations, and cognition and memory. All these problems, with the exception of behavior, steadily increase as the disease progresses and would be useful components of an outcome measure for disease progression (Thompson et al., 2013). However, mood and behavioral problems were often an early sign, were problematic for carers, and had some of the greatest 
impact on the carer-people with prion disease relationship. These symptoms tended to peak in severity in the moderate stage of disease progression, then decrease as the disease progressed and communication and movement became more difficult (Thompson et al., 2014). This symptom domain would not be a useful progression measure, but could be monitored with a specific tool (Thompson et al., 2014).

We acknowledge some limitations of this work. We focused on the difficulties that carers experience in management of the neurodisability caused by prion disease and did not specifically address palliative/end of life care. As the inherited prion diseases have clinical durations many times longer than sporadic CJD, the subtypes of the disease are similarly prevalent in the community, despite the much lower incidence of the familial type. Therefore, our study is representative of the prevalence of prion diseases but not the incidence. There are also some special considerations. For the four people with IPD, the impact of their symptoms on family carers may be amplified by the fact that they have witnessed similar symptoms in other family members, and the carer themselves may be at-risk of developing the disease later in life. We didn't collect data on this possibility. In sporadic CJD, late diagnosis and rapidity of progression may mean that the only appropriate management is end of life care, in which case, many of the recommendations in this paper are not relevant. All the study participants were white. Ethnicity and cultural experiences are likely to affect the experience of caring for people with prion disease and its impact. Finally, comparative data with related neurodegenerative diseases/dementias would be very interesting. These issues are worthy of future study.

Practice nurses and therapists need to help liaison between families and care staff visiting the home, as the staff will often have no experience of caring for a person with dementia and almost certainly no experience of prion disease. Clinicians need to help manage family or carers' expectations regarding care staff and the limitations of their practice. Due to the rapidity of progression of prion disease, a referral to health and social care for home care support should be made early in the expectation of quick deterioration. The introduction of care staff to assist with personal care must be done gradually. Depending on the number of calls, a core of three to five carers is ideal, so people with prion disease and carers can build up a rapport. Ideally carers should be introduced one at a time, with each carer visiting two or three times for a social visit to develop the relationship, before personal care is attempted. A detailed consultation with the family on the person's usual routine and products they use should be undertaken. Documents such as This is Me produced by the UK Alzheimer's Society can be useful (Alzheimer's Society, 2013). Home carers might also attend a training session on prion disease provided by specialist nurses.

People with prion disease often need to be referred to local occupational and physiotherapists for specialist input regarding equipment, adaptations, and exercise. An early referral to Occupational Therapy may be able to set up recorded prompts for people who will be on their own for long periods of time. Having a chalk or white board, or large calendar detailing activities, can provide a helpful visual cue, as can signs and pictures on cupboards, wardrobes, and in rooms. As with other dementias, people can be more independent in familiar environments. Clocks with visual cues, such as a "sun" and "moon" to show night and day, can be helpful. Family carers should consider using telecare technology to alert if someone wakes in the night or wanders from the home. Phone monitors can prevent affected people becoming vulnerable to scam callers, prevent them from calling wrong numbers, or making repeated phone calls. Where possible, people should be encouraged to engage in purposeful activity and continue to perform household tasks, as this will maintain skills and help the person's selfesteem. Careful negotiation with family carers is required as family members can become frustrated with poorly completed tasks or take tasks away from people altogether.

Incontinence was identified as a particular problem in the early stages of prion dementia when the person is still self-caring. Reviewing clothing can help, such as replacing zips and buttons with Velcro or elasticated waistbands. Colored toilet seats can help a person see the toilet more easily and position themselves correctly. Wet wipe-style tissues are better than dry, and limiting the amount available so toilets don't become blocked is a useful idea. Building toilet trips into the natural rhythm of the day, such as before meals and outings, can help to avoid emergencies. Approaches such as, "I'm going to go to the loo, do you want to go too?" can be helpful.

For communication problems, early referrals to a Speech and Language Therapist (SLT) can help in assisting speech maintenance. Practitioners can help family carers by encouraging them to put together homemade picture boards with photos from the home, such as "toilet" and "food." Again documents, such as This is $M e$, or scrapbooks detailing a person's life and achievements, with photographs of key friends and family members, can be a useful tool for families to maintain communication. As in other dementias, distant but salient memories are often preserved in advanced prion disease. A scrapbook can also be a way for visiting care staff to 
engage with people with prion disease and establish relationships and common interests. Training for family and paid carers on recognition of non-verbal cues is useful. Miming actions, using closed questions when offering choices, and sticking to one subject at a time is helpful, as is minimizing distractions such as the television. Speaking to a person one on one, at the same height, and directly facing the person is important.

SLT referral is also useful in managing swallowing difficulties. The usual methods for managing impaired swallowing, such as thickened fluids and soft diet, were effective, with input from SLTs. Timely conversations regarding artificial feeding were essential. Careful education of people with prion disease and their family regarding the implications of swallowing in advanced stages of disease is needed, as chest infections are a common cause of mortality in people with prion disease.

The behavioral symptoms of dementia can have a great effect on carer burden, and carer stress is linked to higher incidence of neuropsychiatric symptoms (Thompson et al., 2014). Practitioners need to work with family carers, so they understand that rude or aggressive behavior is not personal, and the person is not being deliberately "naughty." Challenging behavior is understood today as a form of communication of an unmet need in persons with dementia, and carers should be supported to look for triggers using tools such as ABC charts. Untreated chronic pain can cause agitation, and regular low dose paracetamol has been shown to reduce this behavior in persons with dementia (Husebo et al., 2011). Sudden changes in condition may indicate delirium, and practitioners should ensure carers are aware of delirium precipitating problems, such as UTI and constipation, and encourage them to contact their GP for review. Boredom is often a cause of agitation so a "tool box" of activities is useful, as is playing the person's favorite music.

Further analysis is required in order to better understand the experience of caring for a person with prion disease. Much research into caring for a person with dementia has been carried out using quantitative approaches, which may not be suitable for complex health needs and intervention (Grypdonck, 2006). A qualitative approach, concerned with subjective meanings, can better understand the perceptions and experiences of the carers of dementia; what their needs are and how these change. In this paper, we have summarized and analyzed carer experiences and interpreted these both with respect to disease types and stages, the development of outcome measures, and in care planning. Future work might consider tools to help prediction of care needs, and measurement of carer burden and quality of life using commonly used tools for comparison with other neurodegenerative diseases.

\section{Conflict of interest}

JC is a director and shareholder of D-Gen Ltd., an academic spin-out company working in the field of prion disease diagnosis, decontamination, and therapeutics. No other author has a conflict of interest.

\section{Study Funding}

This work was funded by the Medical Research Council (UK) and the Department of Health (England) and undertaken at UCLH/UCL, who received a proportion of funding from the Department of Health's NIHR Biomedical Research Centre's funding scheme. The Medical Research Council (UK) and the Department of Health funded the PRION-1 trial (G0400713).

Simon Mead and John Collinge are NIHR Senior Investigators.

\section{Description of author's roles}

LF conducted the structured interviews and drafted the manuscript under supervision of $M G$ and $K R$. $\mathrm{PR}, \mathrm{JC}$, and SM were involved in the set up of the study and reviewed and edited the manuscript. SM takes overall responsibility for the paper, drafted responses to reviewers, and did the statistical analysis.

\section{Acknowledgments}

We thank all patients and families for their contribution to this research. We also gratefully acknowledge the contribution of current and past members of the National Prion Clinic, particularly Drs. Steve Wroe, Andrew Thompson, Ana Lukic, Tom Webb, Suvankar Pal, Durre Siddique, Dilip Gajulapalli, Diego Kaski, and Christopher Carswell.

\section{References}

Alzheimer's Society. (2013). This is me-support tool to enable person-centred care. https://www.alzheimers.org.uk/ info/20113/publications_about_living_with_dementia/ 415/this_is_me.

Bucks, R. S., Ashworth, D. L., Wilcock, G. K. and Siegfried, K. (1996). Assessment of activities of daily living in dementia: development of the Bristol activities of daily living scale. Age Ageing, 25, 113-120. doi: 10.1093/ageing/ 25.2.113. 
Clarke, V. and Braun, V. (2017). Thematic analysis. Fournal of Positive Psychology, 12, 297-298. doi: 10.1080/ 17439760.2016 .1262613$.

Cohen-Mansfield, J. (2018). Non-pharmacological interventions for persons with dementia: what are they and how should they be studied? International Psychogeriatrics, 30, 281-283. doi: 10.1017/S104161021800039X.

Cole, L., Samsi, K. and Manthorpe, J. (2018). Is there an "optimal time" to move to a care home for a person with dementia? A systematic review of the literature. International Psychogeriatrics, 1-22. doi: 10.1017/ S1041610218000364.

Collinge, J. (2001). Prion diseases of humans and animals: their causes and molecular basis. Annual Review of Neuroscience, 24, 519-550. doi: 10.1146/annurev.neuro.24.1.519.

Elmir, R., Schmied, V., Jackson, D. and Wilkes, L. (2011). Interviewing people about potentially sensitive topics. Nursing Research, 19, 12-16. doi: 10.7748/nr2011. 10.19.1.12.c8766.

Greenhalgh, T. and Taylor, R. (1997). Papers that go beyond numbers (qualitative research). BMF, 315, 740-743. doi: 10.1136/bmj.315.7110.740.

Grypdonck, M. H. E. (2006). Qualitative health research in the era of evidence-based practice. Qualitative Health Research, 16, 1371-1385. doi: 10.1177/1049732306294089.

Higginbottom, G. M. (2004). Sampling issues in qualitative research. Nursing Research, 12, 7-19.

Husebo, B. S., Ballard, C., Sandvik, R., Nilsen, O. B. and Aarsland, D. (2011). Efficacy of treating pain to reduce behavioural disturbances in residents of nursing homes with dementia: cluster randomised clinical trial. BMF, 343, d4065. doi: 10.1136/bmj.d4065.

Lin, M. C., Macmillan, M. and Brown, N. (2012). A grounded theory longitudinal study of carers' experiences of caring for people with dementia. Dementia-International Fournal of Social Research and Practice, 11, 181-197. doi: 10.1177/1471301211421362.

Livingston, G. et al. (2017). Dementia prevention, intervention, and care. Lancet, 390, 2673-2734. doi: 10.1016/S0140-6736(17)31363-6.

Mead, S. et al. (2011). PRION-1 scales analysis supports use of functional outcome measures in prion disease. Neurology, 77, 1674-1683. doi: 10.1212/WNL. Ob013e3182364890.
Nasreddine, Z. S. et al. (2005). The montreal cognitive assessment, MoCA: a brief screening tool for mild cognitive impairment. Fournal of the American Geriatrics Society, 53, 695-699. doi: 10.1111/j.1532-5415.2005. 53221.x.

Pfeffer, R. I., Kurosaki, T. T., Harrah, C. H., Jr., Chance, J. M. and Filos, S. (1982). Measurement of functional activities in older adults in the community. fournal of Gerontology, 37, 323-329. doi: 10.1093/geronj/ 37.3.323.

Sikkes, S. A., de Lange-de Klerk, E. S., Pijnenburg, Y. A., Scheltens, P. and Uitdehaag, B. M. (2009). A systematic review of instrumental activities of daily living scales in dementia: room for improvement. Fournal of Neurology, Neurosurgery, and Psychiatry, 80, 7-12. doi: 10.1136/jnnp. 2008.155838.

Terwee, C. B. et al. (2007). Quality criteria were proposed for measurement properties of health status questionnaires. Fournal of Clinical Epidemiology, 60, 34-42. doi: 10.1016/j. jclinepi.2006.03.012.

Thompson, A. et al. (2014). Behavioral and psychiatric symptoms in prion disease. The American fournal of Psychiatry, 171, 265-274. doi: 10.1176/appi.ajp.2013. 12111460.

Thompson, A. G. et al. (2013). The medical research council prion disease rating scale: a new outcome measure for prion disease therapeutic trials developed and validated using systematic observational studies. Brain, 136, 1116-1127. doi: 10.1093/brain/awt048.

Trevitt, C. R. and Collinge, J. (2006). A systematic review of prion therapeutics in experimental models. Brain, 129, 2241-2265. doi: 10.1093/brain/awl150.

Tuckett, A. G. (2004). Qualitative research sampling: the very real complexities. Nursing Research, 12, 47-61. doi: 10.7748/nr2004.07.12.1.47.c5930.

Uflacker, A., Edmondson, M. C., Onyike, C. U. and Appleby, B. S. (2015). Caregiver burden in atypical dementias: comparing frontotemporal dementia, Creutzfeldt-Jakob disease, and Alzheimer's disease. International Psychogeriatrics, 28, 269-273. doi: 10.1017/ s1041610215001647.

Walker, W. (2011). Hermeneutic inquiry: insights into the process of interviewing. Nursing Research, 18, 19-27. doi: $10.7748 / \mathrm{nr} 2011.01 .18 .2 .19 . c 8281$. 